\title{
Needle Tip Position and Bevel Direction Have No Effect in the Fluoroscopic Epidural Spreading Pattern in Caudal Epidural Injections: A Randomized Trial
}

\author{
Won Kyoung Kwon, ${ }^{1}$ Ah Na Kim, ${ }^{2}$ Pil Moo Lee, ${ }^{1}$ Cheol Hwan Park, ${ }^{1}$ and Jae Hun Kim ${ }^{1}$ \\ ${ }^{1}$ Department of Anesthesiology and Pain Medicine, Konkuk University Medical Center, Konkuk University School of Medicine, \\ Neungdong-ro 120-1, Kwangjin-gu, Seoul 05030, Republic of Korea \\ ${ }^{2}$ Department of Anesthesiology and Pain Medicine, Wondang OK Pain Clinic, 783 Hoguk-ro, Deogyang-gu, Goyang-si, \\ Gyeonggi-do 10461, Republic of Korea
}

Correspondence should be addressed to Jae Hun Kim; painfree@kuh.ac.kr

Received 8 September 2015; Accepted 26 November 2015

Copyright ( 12016 Won Kyoung Kwon et al. This is an open access article distributed under the Creative Commons Attribution License, which permits unrestricted use, distribution, and reproduction in any medium, provided the original work is properly cited.

\begin{abstract}
Background. Caudal epidural steroid injections (CESIs) are an effective treatment for pain. If the injection spreads in a specific pattern depending on the needle position or bevel direction, it would be possible to inject the agent into a specific and desired area. Objectives. We conducted a prospective randomized trial to determine if the needle position and bevel direction have any effect on the epidural spreading pattern in CESI. Methods. Demographic data of the patient were collected. During CESI, the needle position (middle or lateral) and direction (ventral or dorsal) were randomly allocated. Following fluoroscope-guided injection of $4 \mathrm{~mL}$ contrast media and $10 \mathrm{~mL}$ of injectates, the epidural spreading patterns (ventral or dorsal, bilateral or lateral) were imaged. Results. In the 210 CESIs performed, the needle tip position and bevel direction did not influence the epidural spreading patterns at L4-5 and L5-S1 disc levels. A history of Lumbar spine surgery was associated with a significantly limited spread to each disc level. A midline needle tip position was more effective than the lateral position in spreading to the distant disc levels. Conclusions. Neither the needle tip position nor the bevel direction affected the epidural drug spreading pattern during CESI.
\end{abstract}

\section{Background}

A caudal epidural steroid injection (CESI) has been shown to be an effective treatment approach for low back and/or lower extremity pain [1-4]. Some physicians, including the authors of this paper, tend to position the needle tip at the patient's painful side (right or left) to ensure an effective drug spread to the target area of the pain. Using this method, physicians expect that most of the injected drug will spread to the painful side. It is difficult to determine whether this is true due to the lack of studies on the epidural spreading patterns related to needle tip position in CESI.

As spinal discs are located near the ventral epidural space, ventral spread during CESI could be logically presumed to be an effective treatment in patients who have a herniated intervertebral disc (HIVD) or discogenic pain $[2,5,6]$. Gupta et al. [7] and Ackerman III and Ahmad [8] reported that ventral spread of epidurography was associated with improvements in the pain score after interlaminar, transforaminal, and/or caudal epidural injections.

The objective of this study was to determine the majority of the injectate spread in the ventral epidural space if the bevel of the needle is pointed down. If the agent tends to distribute in a certain pattern according to the needle position or bevel direction, it may be possible to inject the agent into a specific area. We tested the hypothesis that the needle position or bevel direction relates to the pattern of epidural spread during CESI.

\section{Materials and Methods}

This prospective randomized study was performed after gaining ethics approval from the Institutional Review Board at our hospital. After obtaining written informed consent, patients 


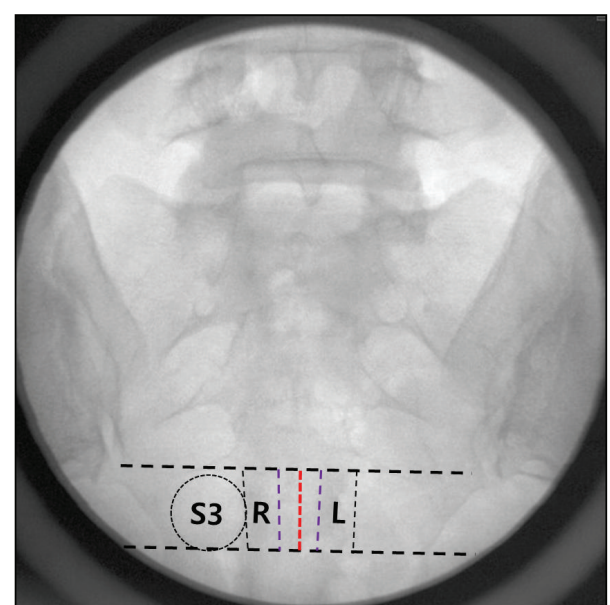

FIgURE 1: Diagram of the needle tip positions used in this study. S3: ventral foramen of S3, R: needle tip position when injecting in the right side, L: needle tip position when injecting in the left side, red dotted line: needle tip position of midline.

scheduled for CESI were included in this study. We collected information regarding age, gender, height, weight, underlying diseases, and any history of lumbar spinal surgery. Exclusion criteria were a lack of informed consent, patients less than 20 years of age, coagulopathy, pregnancy, active infection or pressure sore at the sacral hiatus, or a history of allergic reaction to injected drugs for CESI or any intravascular injection during a procedure.

2.1. Description of Interventions. Using a concealed random number table, the needle position (middle or lateral side) and bevel direction (ventral or dorsal) of the needle tip were each randomly allocated. If the lateral side was selected, the needle was inserted into the patient's more painful side (either right or left; Figure 1). All CESIs were performed by the same experienced physician, with over $8000 \mathrm{C}$-arm fluoroscopy-guided procedures completed. During the CESI, patients were instructed to lie in the prone position, with a $12 \mathrm{~cm}$ pillow under the lower abdomen, and were situated so that the median sacral crest of the S2 and S3 levels was midline of the sacrum in fluoroscopic anteroposterior (AP) view. After local infiltration anesthesia at the injection site, an 18 G Tuohy needle (Tae-Chang Industrial Co., Kongju, Korea) was inserted into the epidural space through the sacral hiatus under fluoroscopic guidance. If the middle needle position was selected (group M), the needle was inserted at the midline of the sacral vertebral body (median sacral crest) (Figure 1). If the lateral side needle position was selected (group L), the needle was inserted into the lateral one-third of the sacral canal by fluoroscopic AP view (Figure 1). The needle tip was advanced at the S3 anterior foramen level (Figure 1). Alternatively, if the S3 anterior foramen was not visible, the needle tip was inserted just below the virtual line between both distal ends of the sacroiliac joint.

Next, the physician turned the bevel of the needle to the selected direction, ventral (group D) or dorsal (group $\mathrm{U})$. After checking for blood aspiration, $4 \mathrm{~mL}$ of contrast medium was injected. If intravascular spreading was noted, the patient was excluded from the study, because some of the injected contrast media was not located in the epidural space. After injection of the contrast media, fluoroscopic AP and lateral views of the L2-3, L3-4, L4-5, and L5-S1 disc levels, and the sacral level were obtained and the images were saved (Figures 2(a) and 2(b)). Subsequently, $10 \mathrm{~mL}$ of injectates (0.125\% levobupivacaine with $2.5-5 \mathrm{mg}$ of dexamethasone and $3 \mathrm{~mL}$ of contrast media) was injected, fluoroscopic AP and lateral views were obtained, and these images were saved (Figure 2(c)).

To evaluate the distribution pattern of the injection, the spread of the injected contrast and drugs was confirmed using the fluoroscopic images. The spreading pattern at L45 and L5-S1 disc levels was determined in relation to the ventral or dorsal side and left or right side. In addition, the maximum distribution was determined. The spreading pattern in each patient was analyzed by the same experienced pain physician and radiologist, neither of whom was involved in the procedure. If the two physicians did not agree on the results of the analysis, an additional experienced pain physician assessed the fluoroscopic images, and a consensus was reached.

2.2. Sample Size Justification. This study was powered to detect a difference in the epidural spreading pattern according to the needle position. In the preliminary investigation, the rate of bilateral spreading at the L5-S1 level was $90 \%$ (18 of 20 CESIs) in the central position group and 75\% (15 of 20 CESIs) in the lateral position group. To obtain $80 \%$ power for analysis, $97 \mathrm{CESIs}$ were required in each group. The only attrition was among patients who received intravascular injection, and the study was halted when the lateral group reached 100 cases.

2.3. Statistical Methods. Mean differences in age, height, and weight were analyzed by Student's $t$-test. Proportional differences in gender, lumbar spinal surgery history, needle position, and bevel direction were analyzed by the chi square test. Possible factors affecting the epidural spread, such as bevel direction, needle position, and history of lumbar spine operation, were analyzed by logistic regression analysis. $P$ values of less than 0.05 were considered statistically significant.

\section{Results}

There were 229 CESIs performed in this study; 19 cases $(8.3 \%)$ were excluded because they received an intravascular injection during the CESI. Therefore, 210 CESIs (174 patients) were included in this study. Following the injection of $14 \mathrm{~mL}$ of injectates, bilateral epidural spreading occurred in $83.8 \%$ of patients (124 of 148) at the L4-5 disc level and $85.4 \%$ of patients (170 of 199) at the L5-S1 disc level (Table 1). Ventral epidural spreading occurred in $63.5 \%$ of patients (94 of 148) at the L4-5 disc level, increasing to $74.9 \%$ of patients (149 of 199) at the L5-S1 disc level.

Needle position comparison was performed between groups M (110 patients) and L (100 patients). There were 


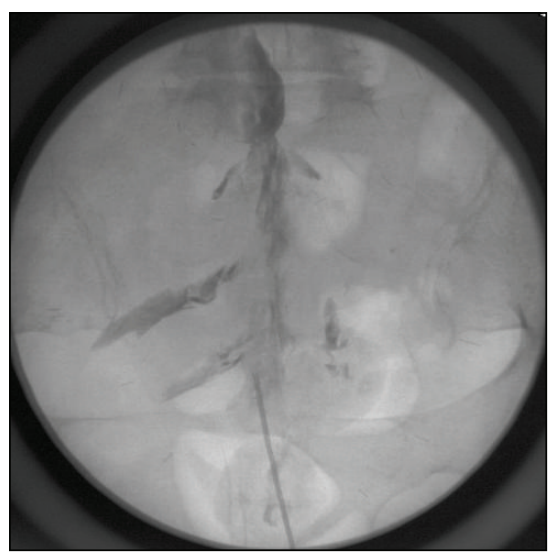

(a)

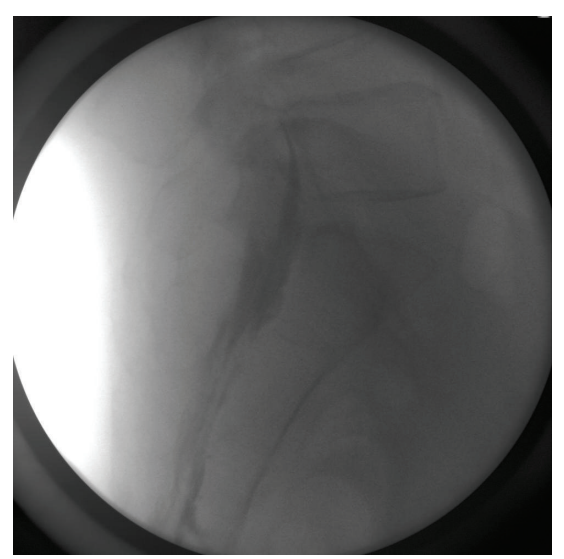

(b)

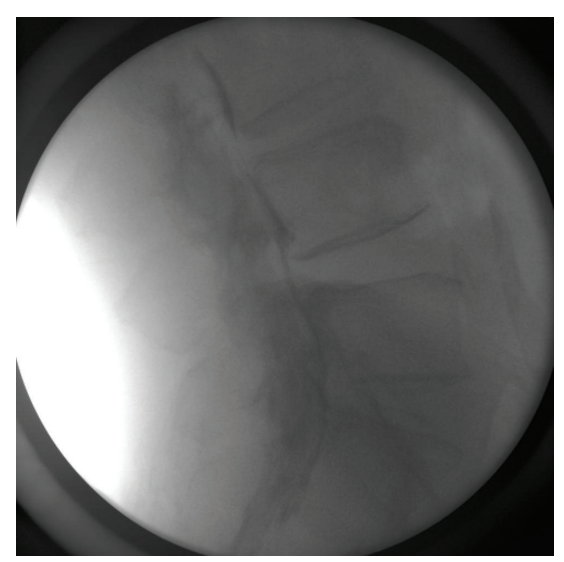

(c)

FIGURE 2: Sample image of the epidural spreading pattern after injection of $4 \mathrm{~mL}$ of contrast media using the left side needle tip position ((a) anteroposterior view; (b) lateral view) and (c) after injection of $14 \mathrm{~mL}$ of injectates.

TABLE 1: The rates of bilateral and ventral drug spread at L4-5 and L5-S1 disc levels after injection of $4 \mathrm{~mL}$ of contrast medium or $14 \mathrm{~mL}$ of injectates.

\begin{tabular}{lcccc}
\hline \multirow{2}{*}{ Injection drug } & \multicolumn{2}{c}{ Bilateral spread } & \multicolumn{2}{c}{ Ventral spread } \\
& L5-S1 level & L4-5 level & L5-S1 level & L4-5 level \\
\hline $4 \mathrm{~mL}$ of contrast & $115 / 175(65.7 \%)$ & $35 / 56(62.5 \%)$ & $101 / 175(57.7 \%)$ & $24 / 56(42.9 \%)$ \\
$14 \mathrm{~mL}$ of injectates & $170 / 199(85.4 \%)$ & $124 / 148(83.8 \%)$ & $149 / 199(74.9 \%)$ & $94 / 148(63.5 \%)$ \\
\hline
\end{tabular}

The data is expressed as "the number of cases with spreading/total number of cases" (percent of cases with spreading).

TABLE 2: Demographic data of the patients treated in group $M$ and group L.

\begin{tabular}{lccc}
\hline & $\begin{array}{c}\text { Group M } \\
(n=110)\end{array}$ & $\begin{array}{c}\text { Group L } \\
(n=100)\end{array}$ & $P$ value \\
\hline Age & $63.4 \pm 14.9$ & $59.8 \pm 16.0$ & 0.092 \\
Gender (female/male) & $73 / 37$ & $55 / 45$ & 0.092 \\
Height & $159.8 \pm 8.4$ & $161.0 \pm 10.5$ & 0.383 \\
Weight & $61.0 \pm 10.6$ & $62.1 \pm 11.8$ & 0.438 \\
Lumbar spine operation history & 32 & 18 & 0.059 \\
DM & 17 & 8 & 0.096 \\
HTN & 49 & 37 & 0.267 \\
\hline
\end{tabular}

Individuals from group $\mathrm{M}$ had their needle inserted at the midline of the sacral vertebral body.

Individuals from group $\mathrm{L}$ had their needle inserted into the lateral one-third of the sacral canal.

Data is expressed as "mean $\pm \mathrm{SD}$ " or "number of individuals."

DM: diabetes mellitus; HTN: hypertension.

no significant differences between the two groups regarding age, gender, height, weight, and lumbar spine surgery history (Table 2). There were also no differences between the two groups regarding the pattern of bilateral epidural spreading (right and left side) or ventral epidural spreading (Table 3). In group M, bilateral epidural spreading after injection of $14 \mathrm{~mL}$ of injectates was observed in $84.8 \%$ of patients (67 of 79) at the L4-5 disc level and in $87.5 \%$ of patients (91 of 104) at the L5-S1 disc level (Table 3). Ventral epidural spreading after injection of $14 \mathrm{~mL}$ of injectates was observed in $68.4 \%$ of patients ( 54 of 79 ) at the L4-5 disc level and in $76.9 \%$ of patients (80 of 104) at the L5-S1 disc level. There were no statistical differences observed between the epidural spreading to the right, left, or both sides for each needle position (right, middle, and left).

Needle bevel direction comparison was performed between groups U (99 patients) and D (111 patients). There were no significant differences observed regarding age, gender, height, weight, and lumbar spine surgery history between the two groups (Table 4). There were also no differences between the two groups regarding the pattern of bilateral epidural spreading (right and left side) or ventral epidural spreading (Table 5). After caudal epidural injection of $14 \mathrm{~mL}$ of injectates, spreading occurred in 59 patients $(28.1 \%)$ to the L2-3 disc level, 99 patients (47.1\%) to the L3-4 disc level, 148 patients $(70.5 \%)$ to the L4-5 disc level, and 199 patients (94.8\%) to the L5-S1 disc level. There were no statistical differences in epidural spreading on the ventral, dorsal, or bilateral sides for each bevel direction (ventral or dorsal).

Using logistic regression analysis, the needle bevel direction (group $U$ or group D) did not correlate to the drug spreading to the L2-3, L3-4, L4-5, and L5-S1 disc levels (Figure 3). The degree of drug spreading to the L2-3 and L34 disc levels was significantly higher in group $\mathrm{M}$ compared to group $\mathrm{L}(P<0.05)$ (Figures 3(a) and 3(b)). The degree of drug spreading at the L2-3, L3-4, L4-5, and L5-S1 disc levels in patients without a history of lumbar spine surgery was significantly higher than that in patients with a history of lumbar spine surgery $(P<0.05)$ (Figure 3$)$. 


\begin{tabular}{lcccc|c|c|}
\hline & $P$ value & Odds ratio & $95 \% \mathrm{CI}$ & & \\
\hline Bevel direction & 0.365 & 0.751 & $0.404-1.396$ & $1.170-4.194$ & & 1 \\
Midline position & 0.015 & 2.216 & $0.151-0.819$ & 0.1 & Odds ratio \\
Spine operation & 0.015 & 0.352 & & & 10 \\
\hline
\end{tabular}

(a)

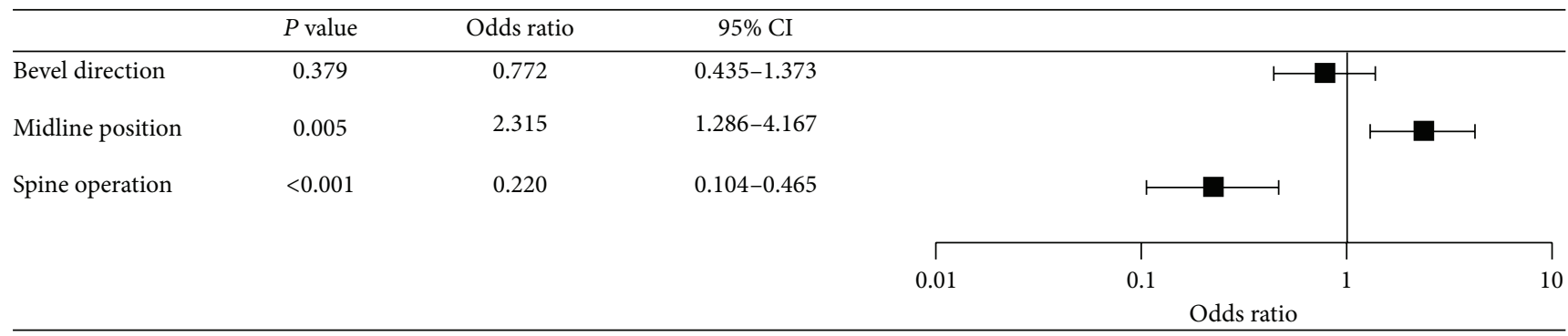

(b)

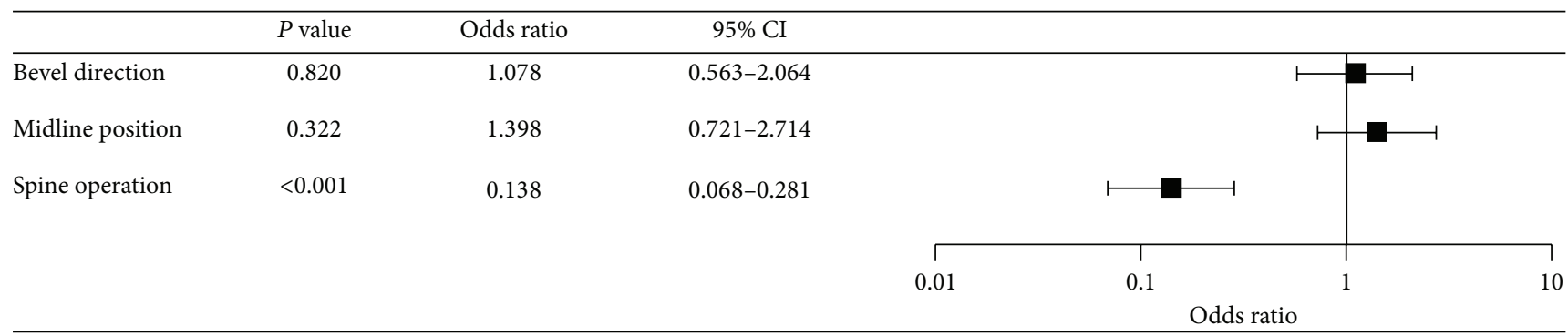

(c)

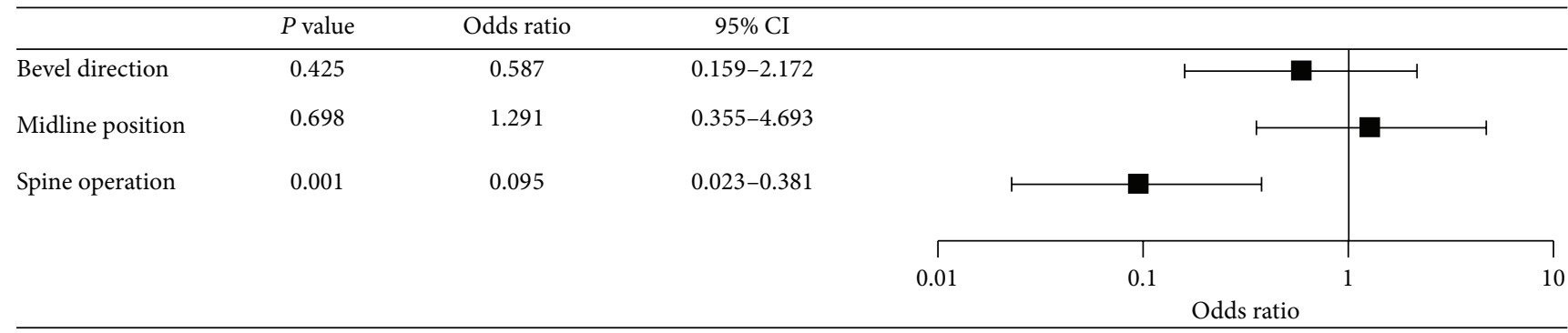

(d)

FIGURE 3: Logistic regression analysis of the epidural spread at (a) L2-3, (b) L3-4, (c) L4-5, and (d) L5-S1 disc levels after injection of 14 mL of injectates.

\section{Discussion}

In the present study, the needle tip position (midline or lateral) during CESI was not related to the epidural spreading pattern at the L4-5 and L5-S1 disc levels. The needle tip position to either the right or left side also did not relate to the epidural spread at the L4-5 and L5-S1 disc levels. Injections in all needle tip positions (right, middle, and left) were characterized by the majority of the drug spreading bilaterally at both the L4-5 and L5-S1 disc levels. Therefore, despite the needle tip being positioned on the lateral side in a patient who has pain in only one side, the epidural spread will most likely be bilateral, rather than unilateral only on the painful side, at the L4-5 and L5-S1 disc levels. These results were inconsistent with our hypothesis that if the needle tip is positioned at a specific side during CESI, the injectate will spread preferentially on that specific side. Lee et al. conducted a study of 22 cases of caudal epidurography following injection of $5 \mathrm{~mL}$ of contrast media conducted with the needle positioned toward the affected side in CESI [9]. In contrast to the present study, Lee et al. determined that the average ratio of the contrast media area on the affected side to the total area of epidurography was $73.5 \%$, as measured by pixel count within the area of epidurography [9]. Additionally, they reported 
TABLE 3: The rates of bilateral and ventral epidural spread in groups $\mathrm{M}$ and $\mathrm{L}$ after injection of $4 \mathrm{~mL}$ of contrast medium or $14 \mathrm{~mL}$ of injectates.

\begin{tabular}{|c|c|c|c|c|}
\hline Injection drug & Epidural spreading & Group M $(n=110)$ & Group L $(n=100)$ & $P$ value \\
\hline \multirow{4}{*}{$4 \mathrm{~mL}$ of contrast medium } & Bilateral spreading at L5/S1 & $66 / 93(71.0 \%)$ & $49 / 82(59.8 \%)$ & 0.119 \\
\hline & Bilateral spreading at L4/5 & $21 / 32(65.6 \%)$ & $14 / 24(58.3 \%)$ & 0.577 \\
\hline & Ventral spreading at L5/S1 & $58 / 93(62.4 \%)$ & $43 / 82(52.4 \%)$ & 0.185 \\
\hline & Ventral spreading at L4/5 & $17 / 32(53.1 \%)$ & $7 / 24(29.2 \%)$ & 0.073 \\
\hline \multirow{4}{*}{$14 \mathrm{~mL}$ of injectates } & Bilateral spreading at L5/S1 & $91 / 104(87.5 \%)$ & $79 / 95(83.2 \%)$ & 0.386 \\
\hline & Bilateral spreading at $\mathrm{L} 4 / 5$ & $67 / 79(84.8 \%)$ & $57 / 69(82.6 \%)$ & 0.717 \\
\hline & Ventral spreading at L5/S1 & $80 / 104(76.9 \%)$ & $69 / 95(72.6 \%)$ & 0.486 \\
\hline & Ventral spreading at $\mathrm{L} 4 / 5$ & $54 / 79(68.4 \%)$ & $40 / 69(58.0 \%)$ & 0.191 \\
\hline
\end{tabular}

Individuals from group $\mathrm{M}$ had their needle inserted at the midline of the sacral vertebral body.

Individuals from group L had their needle inserted into the lateral one-third of the sacral canal.

The data is expressed as "the number of cases with spreading/total number of cases" (percent of cases with spreading).

TABLE 4: Demographic data of the patients treated in group $U$ and group D.

\begin{tabular}{lccc}
\hline & $\begin{array}{c}\text { Group U } \\
(n=99)\end{array}$ & $\begin{array}{c}\text { Group D } \\
(n=111)\end{array}$ & $P$ value \\
\hline Age & $60.5 \pm 16.7$ & $62.7 \pm 14.4$ & 0.313 \\
Gender (female/male) & $61 / 38$ & $67 / 44$ & 0.852 \\
Height & $160.8 \pm 10.2$ & $160.1 \pm 8.8$ & 0.576 \\
Weight & $62.6 \pm 9.9$ & $60.5 \pm 12.2$ & 0.199 \\
Lumbar spine operation history & 24 & 26 & 0.889 \\
DM & 13 & 12 & 0.604 \\
HTN & 38 & 48 & 0.475 \\
\hline
\end{tabular}

Individuals from group $\mathrm{U}$ had the bevel of the needle headed to the dorsal side.

Individuals from group D had the bevel of the needle headed to the ventral side.

Data is expressed as "mean \pm SD" or "number of individuals."

DM: diabetes mellitus; HTN: hypertension.

that $68.2 \%$ of the patients showed a predominant spread to the needle side, $31.8 \%$ showed nonpredominant spreading, and $13.6 \%$ showed greater spreading to the opposite side [9]. However, in the predominant spread at each nerve root, there were no statistically significant differences between the needle side and opposite side at the L4, L5, and S1 (S2 and S3 were exceptions) nerve roots. Therefore, the predominant spreading may be limited at the sacrum, such as at the S2 and S3 levels described in the results. The number of cases in our study is larger than that of Lee et al.s study. In our study, we did not investigate the spreading pattern in the sacrum, but instead at the L4-5 and L5-S1 disc levels, which are the most common sites of HIVD [10] and spinal stenosis [11]; therefore, CESI is often conducted at these levels.

The bevel direction (ventral or dorsal) was also not related to the epidural spreading pattern at the L4-5 and L5-S1 disc levels. Therefore, positioning the needle upwards or downwards does not indicate that the epidural spreading will occur on only the dorsal or ventral side. In interlaminar epidural injections, the Tuohy needle bevel direction to the cephalad or caudal side does not relate to spread [12-14] or has only minor effects on the spread $[15,16]$. The diameter of the 18-gauge Tuohy needle in this study was $1.2 \mathrm{~mm}$, according to the company brochure. Therefore, the difference of the location of bevel between the up and down side of the needle would be about $1.2 \mathrm{~mm}$. It is believed that this small distance $(1.2 \mathrm{~mm})$ may not influence the rate of spreading to the ventral or dorsal side. Despite the different bevel direction of the needle, there were numerous instances in which the drugs spread to the ventral epidural space at the L4-5 level (group U: 59.4\%, group D: 67.1\%) and the L5-S1 level (group U: $72.6 \%$, group D: $76.9 \%$ ) after injection of $14 \mathrm{~mL}$ of drugs. To inject the drug into the targeted level and side in the epidural space, it was more effective to rotate the Tuohy needle tip $45^{\circ}$ and place the catheter to advance the drug into the desired level in interlaminar epidural injections $[17,18]$. In CESI, needle tip rotation and catheter insertion at the desired level may also be effective for the injectate to approach the target level. Catheter insertion via sacral hiatus, and drug injection at the target site, is usually performed in epidural neuroplasty [19-21].

In this study, a midline needle tip position was related to spreading to distant disc levels. Drug spread at the L23 and L3-4 disc levels was different according to the needle position. If the target level of CESI was at the L2-3 or L34 disc level, a midline needle tip position would be helpful in spreading the drug to the target level. After injection of $14 \mathrm{~mL}$ of injectates, at L4-5 and L5-S1 disc level, a midline or lateral area needle tip position did not affect the spreading of the injectate. It is believed that the $14 \mathrm{~mL}$ injection volume may easily approach the L4-5 and L5-S1 level in most cases, even with a different needle tip position (midline or lateral). Despite the different needle tip positions, bilateral spreading was the most common pattern in CESI. Therefore, we believe that the relatively short distance from the midline needle tip position to a higher spine level of bilateral spreading may have advantages in the degree of drug spread at high disc levels such as L2-3 and L3-4. Lee et al. reported that needle position was related to epidural contrast spreading at the S2 and S3 nerve roots [9]. Therefore, lateral needle tip position may cause leakage through the sacral foramen, and less volume of injectate may spread to the lumbar level rather than with the needle tip in the midline position. We believe that the relatively small volume of injectate in the lateral needle tip position can also reduce the epidural spread to higher levels of the lumbar spine. 
TABLE 5: The rates of bilateral and ventral epidural spread in groups $\mathrm{U}$ and $\mathrm{D}$ after injection of $4 \mathrm{~mL}$ of contrast medium or $14 \mathrm{~mL}$ of injectates.

\begin{tabular}{|c|c|c|c|c|}
\hline Injection drug & Epidural spreading & Group $\mathrm{U}(n=99)$ & Group D $(n=111)$ & $P$ value \\
\hline \multirow{4}{*}{$4 \mathrm{~mL}$ of contrast medium } & Bilateral spreading at L5/S1 & $55 / 83(66.3 \%)$ & $60 / 92(65.2 \%)$ & 0.232 \\
\hline & Bilateral spreading at $\mathrm{L} 4 / 5$ & $16 / 25(64.0 \%)$ & $19 / 31(61.3 \%)$ & 0.835 \\
\hline & Ventral spreading at L5/S1 & $44 / 83(53.8 \%)$ & $57 / 92(62.0 \%)$ & 0.232 \\
\hline & Ventral spreading at $\mathrm{L} 4 / 5$ & $10 / 25(40.0 \%)$ & $14 / 31(45.2 \%)$ & 0.698 \\
\hline \multirow{4}{*}{$14 \mathrm{~mL}$ of injectates } & Bilateral spreading at L5/S1 & $78 / 95(82.1 \%)$ & $92 / 104(88.5 \%)$ & 0.204 \\
\hline & Bilateral spreading at $\mathrm{L} 4 / 5$ & $58 / 69(84.1 \%)$ & $66 / 79(83.5 \%)$ & 0.933 \\
\hline & Ventral spreading at L5/S1 & $69 / 95(72.6 \%)$ & $80 / 104(76.9 \%)$ & 0.486 \\
\hline & Ventral spreading at L4/5 & $41 / 69(59.4 \%)$ & $53 / 79(67.1 \%)$ & 0.334 \\
\hline
\end{tabular}

Individuals from group $\mathrm{U}$ had the bevel of the needle headed to the dorsal side. Individuals from group D had the bevel of the needle headed to the ventral side.

The data is expressed as "the number of cases with spreading/total number of cases" (percent of cases with spreading).

In this study, a history of L-spine surgery was a restrictive factor of drug spread at the L2-3, L3-4, L4-5, and L5-S1 disc levels. Adhesions in the epidural space could be triggered by the postoperative wound healing process and scar tissue formation after spinal surgery and have the potential to limit drug distribution in the epidural space $[19,21,22]$. Therefore, epidural adhesion or scarring in patients with a history of Lspine surgery can decrease the cephalic spreading of the drug.

In patients without a history of L-spine surgery, the rate of spreading of $14 \mathrm{~mL}$ of injectate was $31.9 \%$ at the $\mathrm{L} 2-3,54.5 \%$ at the $\mathrm{L} 3-4,80.6 \%$ at the L4-5, and $98.1 \%$ at the L5-S1 disc levels. After $14 \mathrm{~mL}$ of epidural injection, in the patients who had no history of L-spine surgery, the injected drugs reached the L5S1 disc level in almost all cases and the L4-5 disc level in 80.6\% of cases. Despite the different needle tip position (midline or lateral), in most cases, $14 \mathrm{~mL}$ of CESI epidural drugs can reach the L4-5 and L5-S1 disc levels. Therefore, $14 \mathrm{~mL}$ may be a reasonable volume in CESI if the target is the L4-5 or L5S1 disc level. Kim et al. reported that the epidural spreading level was not significantly changed with subsequent injection from $10 \mathrm{~mL}$ to $50 \mathrm{~mL}$ in 32 patients of CESI [23]. They said that there was no direct relationship between the volume of injected solution and the number of blocked segments due to the anterior leakage of solution through the sacral foramen. Nevertheless, in previous systematic review, the amount of injected fluid in epidural block was correlated with pain relief among the patients with radicular pain and/or low back pain [24]. Investigation of 20 cases of magnetic resonance epidurography after CESI showed the epidural spread level after injection of $10 \mathrm{~mL}$ or $20 \mathrm{~mL}$ of injectate [25]. After injection of $10 \mathrm{~mL}$, the injectate was observed at the L3-4 level (56\%), the L4-5 level (66\%), and the L5-S1 level (89\%) [25]. After injection of $20 \mathrm{~mL}$, the injectate was observed at the L3-4 level in all cases [25]. Although the case number was small (9 cases of $10 \mathrm{~mL}$ injection and 11 cases of $20 \mathrm{~mL}$ injection), the study showed that the volume could affect the highest epidural spreading level in CESI. Although there was no linear correlation between injected volume and spreading level of spine, further investigation about the most effective volume of injectate in CESI will be required.

Cleary et al. reported that a $30^{\circ}$ Trendelenburg tilt from the lumbar spine was more effective for cephalic drug delivery in CESI in order to eradicate lumbar lordosis [26]. In our study, a pillow was positioned under the patient's lower abdomen to reduce the lumbar lordosis. The results of the previous study suggest that the dural surface area is one of the primary determinants of epidural longitudinal distribution of local anesthetics [27]. According to this mechanism, conditions that increase abdominal pressure, such as obesity or pregnancy, compress the dural sac, reduce the dual surface area, and reduce the epidural cavity volume so that the final level of the drug injected is higher when the drug is injected into the epidural space $[27,28]$. Trendelenburg tilt at the lumbar spine, or a pillow under the abdomen, has been related to a decrease in lumbar lordosis and may increase the abdominal pressure, thereby possibly increasing the spread of the injectate at a high level of the spine.

There are some limitations in this study. Although we calculated the sample size, there was no correlation between needle tip position/direction of bevel and epidural spreading pattern. Nevertheless, we believe that with no correlation in 210 cases, our hypothesized relation between needle tip position or bevel direction and epidural spreading pattern that was tested in this study is likely not useful in clinical cases.

In conclusion, neither the needle tip position (midline or lateral side) nor the Tuohy needle bevel direction impact the epidural drug spreading pattern at the L4-5 and L5-S1 disc levels during CESI. Fourteen milliliters is a reasonable total volume of injectate for the L4-5 or L5-S1 disc pathology in CESI. Patients with a history of L-spine surgery may experience limited epidural spreading to most lumbar disc levels. However, a midline needle tip position may be helpful in epidural drug spreading to the distant disc levels in CESI.

\section{Additional Points}

The authors conducted a prospective randomized trial to determine if the needle position and bevel direction have any effect on the epidural spreading pattern in caudal epidural steroid injections (CESIs). In the 210 CESIs performed, neither the needle tip position nor the bevel direction affected the epidural drug spreading pattern during CESI. Patients with a history of lumbar spine surgery may experience 
limited epidural spreading to the different lumbar disc levels. A midline needle tip position may promote epidural drug spreading to the distant disc levels in CESI.

\section{Competing Interests}

The authors declare that they have no competing interests.

\section{References}

[1] L. Manchikanti, V. Singh, V. Pampati, F. J. E. Falco, and J. A. Hirsch, "Comparison of the efficacy of caudal, interlaminar, and transforaminal epidural injections in managing lumbar disc herniation: is one method superior to the other?" Korean Journal of Pain, vol. 28, no. 1, pp. 11-21, 2015.

[2] S. P. Cohen, M. C. Bicket, D. Jamison, I. Wilkinson, and J. P. Rathmell, "Epidural steroids: a comprehensive, evidence-based review," Regional Anesthesia and Pain Medicine, vol. 38, no. 3, pp. 175-200, 2013.

[3] J. G. Leem, "Epidural steroid injection: a need for a new clinical practice guideline," Korean Journal of Pain, vol. 27, no. 3, pp. 197199, 2014.

[4] L. Manchikanti, P. S. Staats, D. E. Nampiaparampil, and J. A. Hirsch, "What is the role of epidural injections in the treatment of lumbar discogenic pain: a systematic review of comparative analysis with fusion," Korean Journal of Pain, vol. 28, no. 2, pp. 75-87, 2015.

[5] A. T. Parr, L. Manchikanti, H. Hameed et al., "Caudal epidural injections in the management of chronic low back pain: a systematic appraisal of the literature," Pain Physician, vol. 15, no. 3, pp. E159-E198, 2012.

[6] J. H. Lee, J. H. An, and S.-H. Lee, "Comparison of the effectiveness of interlaminar and bilateral transforaminal epidural steroid injections in treatment of patients with lumbosacral disc herniation and spinal stenosis," Clinical Journal of Pain, vol. 25, no. 3, pp. 206-210, 2009.

[7] R. Gupta, S. Singh, S. Kaur, K. Singh, and K. Aujla, "Correlation between epidurographic contrast flow patterns and clinical effectiveness in chronic lumbar discogenic radicular pain treated with epidural steroid injections via different approaches," Korean Journal of Pain, vol. 27, no. 4, pp. 353-359, 2014.

[8] W. E. Ackerman III and M. Ahmad, "The efficacy of lumbar epidural steroid injections in patients with lumbar disc herniations," Anesthesia and Analgesia, vol. 104, no. 5, pp. 1217-1222, 2007.

[9] J. H. Lee, D. M. Yoon, T. D. Kwon, and K. B. Yoon, “An evaluation of contrast medium spread on caudal epidurography with the needle positioned toward the affected side in patients with unilateral lumbosacral radiculopathy," Skeletal Radiology, vol. 41, no. 12, pp. 1613-1618, 2012.

[10] A. Ali, S. A. Khan, A. Aurangzeb et al., "Lumbar disc herniation in patients with chronic backache," Journal of Ayub Medical College, Abbottabad, vol. 25, no. 3-4, pp. 68-70, 2013.

[11] Y. Ishimoto, N. Yoshimura, S. Muraki et al., "Associations between radiographic lumbar spinal stenosis and clinical symptoms in the general population: the Wakayama Spine Study," Osteoarthritis and Cartilage, vol. 21, no. 6, pp. 783-788, 2013.

[12] P. H. Rosenberg, L. Saramies, and A. Alila, "Lumbar epidural anaesthesia with bupivacaine in old patients: effect of speed and direction of injection," Acta Anaesthesiologica Scandinavica, vol. 25, no. 3, pp. 270-274, 1981.
[13] M. Yokoyama, M. Hanazaki, H. Fujii et al., "Correlation between the distribution of contrast medium and the extent of blockade during epidural anesthesia," Anesthesiology, vol. 100, no. 6, pp. 1504-1510, 2004.

[14] C.-C. Liu, J. Spielberger, W.-Y. Chou, S.-W. Chau, P.-H. Liu, and P.-H. Tan, "Evaluation of the effects of caudal or cephalic epidural catheterization on the characteristics of lumbar epidural anesthesia," Acta Anaesthesiologica Taiwanica, vol. 43, no. 2, pp. 79-83, 2005.

[15] W. Y. Park, K. C. Poon, M. D. Massengale, and T. E. Macnamara, "Direction of the needle bevel and epidural anesthetic spread," Anesthesiology, vol. 57, no. 4, pp. 327-328, 1982.

[16] W. A. Visser, R. A. Lee, and M. J. M. Gielen, "Factors affecting the distribution of neural blockade by local anesthetics in epidural anesthesia and a comparison of lumbar versus thoracic epidural anesthesia," Anesthesia and Analgesia, vol. 107, no. 2, pp. 708-721, 2008

[17] B. Borghi, V. Agnoletti, A. Ricci, H. Van Oven, N. Montone, and A. Casati, "A prospective, randomized evaluation of the effects of epidural needle rotation on the distribution of epidural block," Anesthesia and Analgesia, vol. 98, no. 5, pp. 1473-1478, 2004.

[18] M. Dauri, T. Sidiropoulou, E. Fabbi, P. P. Mariani, and A. F. Sabato, "Intentional lateral epidural catheter placement for anterior cruciate ligament reconstruction," Acta Anaesthesiologica Scandinavica, vol. 49, no. 5, pp. 671-676, 2005.

[19] J. H. Kim, H. J. Jung, F. S. Nahm, and P. B. Lee, "Does improvement in epidurography following percutaneous epidural neuroplasty correspond to patient outcome?" Pain Practice, vol. 15, no. 5, pp. 407-413, 2015.

[20] C. H. Oh, G. Y. Ji, P. G. Cho et al., "The catheter tip position and effects of percutaneous epidural neuroplasty in patients with lumbar disc disease during 6-months of follow-up," Pain Physician, vol. 17, no. 5, pp. E599-E608, 2014.

[21] F. Lee, D. E. Jamison, R. W. Hurley, and S. P. Cohen, "Epidural lysis of adhesions," Korean Journal of Pain, vol. 27, no. 1, pp. 3-15, 2014.

[22] J. Devulder, L. Bogaert, F. Castille, A. Moerman, and G. Rolly, "Relevance of epidurography and epidural adhesiolysis in chronic failed back surgery patients," Clinical Journal of Pain, vol. 11, no. 2, pp. 147-150, 1995.

[23] K.-M. Kim, H.-S. Kim, K.-H. Choi, and W.-S. Ahn, "Cephalic spreading levels after volumetric caudal epidural injections in chronic low back pain," Journal of Korean Medical Science, vol. 16, no. 2, pp. 193-197, 2001.

[24] D. L. Rabinovitch, A. Peliowski, and A. D. Furlan, "Influence of lumbar epidural injection volume on pain relief for radicular leg pain and/or low back pain," Spine Journal, vol. 9, no. 6, pp. 509-517, 2009.

[25] D. T. Murphy, E. C. Kavanagh, A. Poynton, V. O. Chan, M. R. Moynagh, and S. Eustace, "MR epidurography: distribution of injectate at caudal epidural injection," Skeletal Radiology, vol. 44, no. 4, pp. 565-571, 2015.

[26] M. Cleary, C. Keating, and A. R. Poynton, "The flow patterns of caudal epidural in upper lumbar spinal pathology," European Spine Journal, vol. 20, no. 5, pp. 804-807, 2011.

[27] H. Higuchi, Y. Adachi, and T. Kazama, "Factors affecting the spread and duration of epidural anesthesia with ropivacaine," Anesthesiology, vol. 101, no. 2, pp. 451-460, 2004.

[28] Q. H. Hogan, R. Prost, A. Kulier, M. L. Taylor, S. Liu, and L. Mark, "Magnetic resonance imaging of cerebrospinal fluid volume and the influence of body habitus and abdominal pressure," Anesthesiology, vol. 84, no. 6, pp. 1341-1349, 1996. 


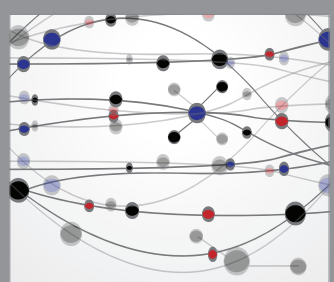

The Scientific World Journal
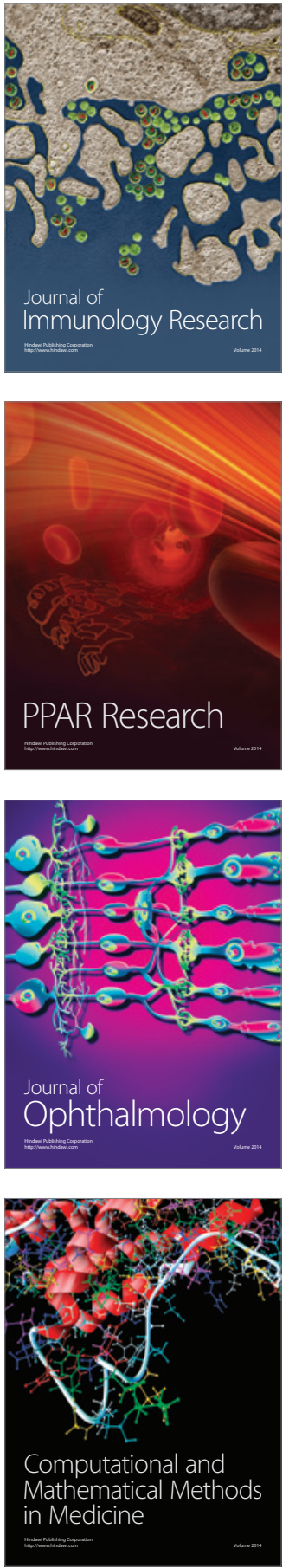

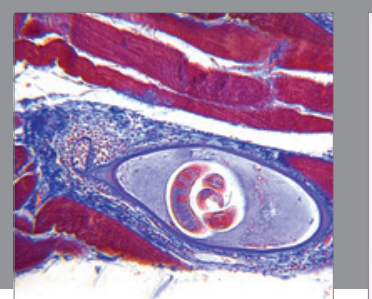

Gastroenterology Research and Practice

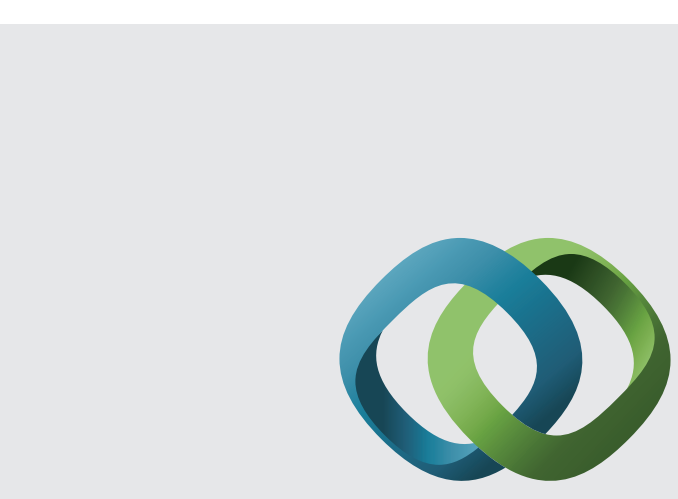

\section{Hindawi}

Submit your manuscripts at

http://www.hindawi.com
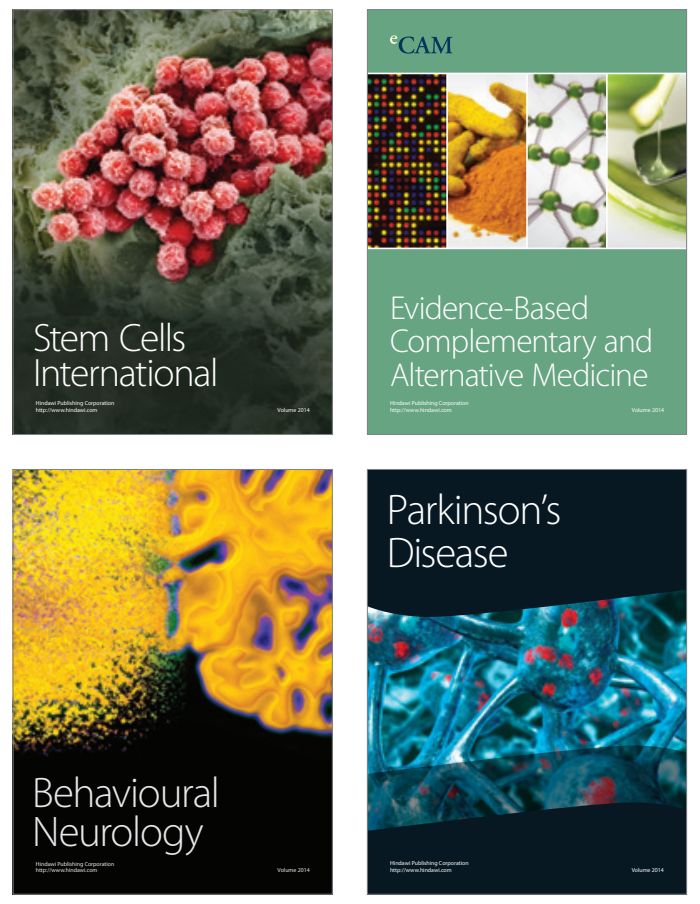
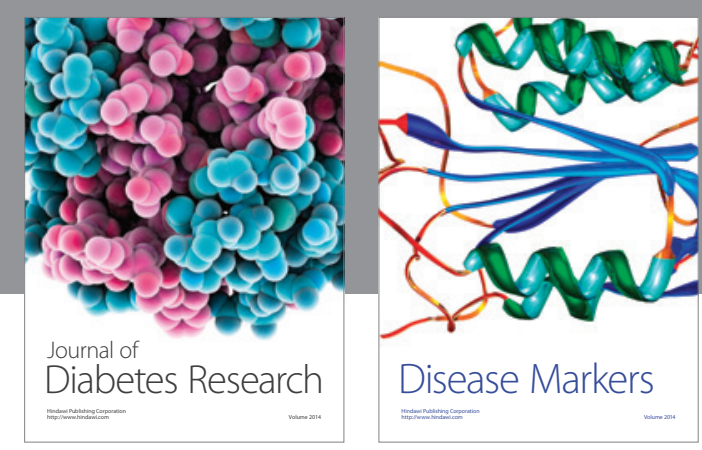

Disease Markers
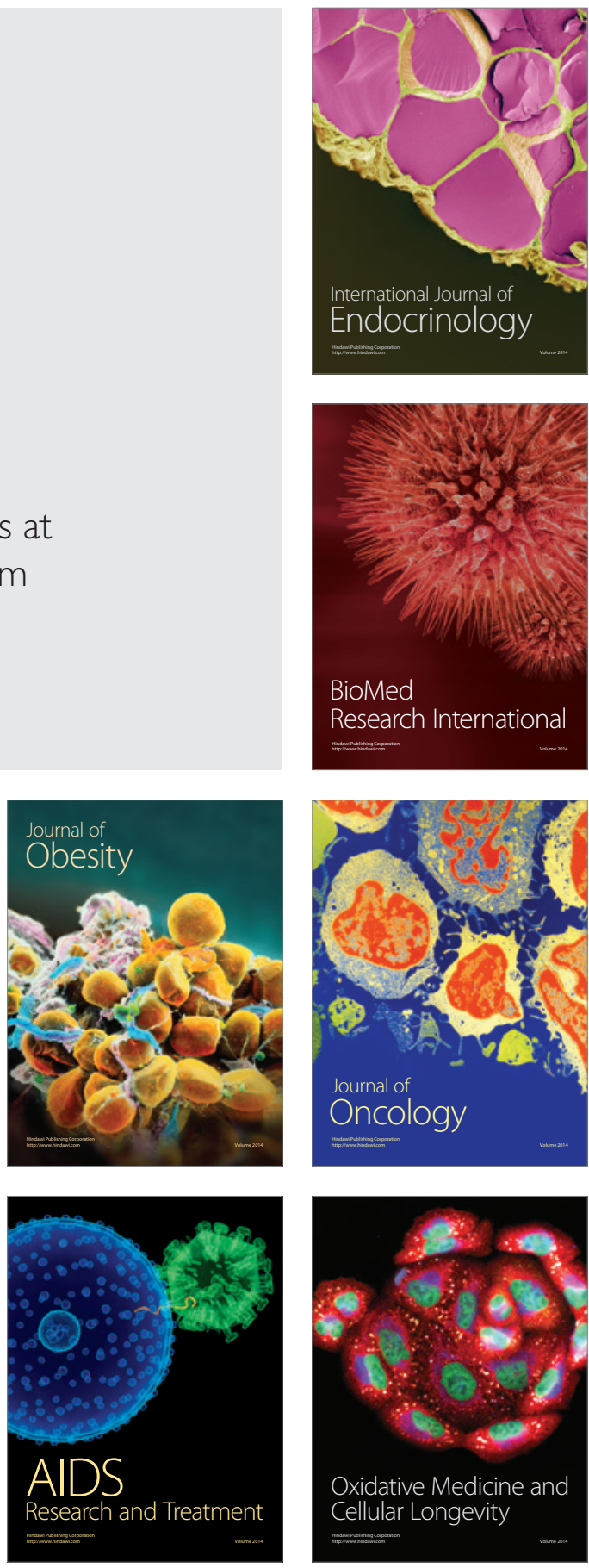